\title{
BMJ Open Diagnostic, preventive and therapeutic evidence in obstetrics for the implementation of patient blood management: a systematic review protocol
}

Iosief Abraha, ${ }^{1,2}$ Alessandro Montedori, ${ }^{2}$ Gian Carlo Di Renzo, ${ }^{3}$ Patrizio Angelozzi, ${ }^{4}$ Marta Micheli, ${ }^{5}$ Danilo Carloni, ${ }^{6}$ Antonella Germani, ${ }^{5}$ Gianluca Palmieri, ${ }^{7}$ Marta Casali, ${ }^{8}$ Chiara Maria Grazia Nenz, ${ }^{9}$ Elisabetta Gargano, ${ }^{10}$ Michela Pazzaglia, ${ }^{11}$ Laura Berchicci, ${ }^{12}$ Simonetta Tesoro, ${ }^{13}$ Giorgio Epicoco, ${ }^{14}$ Gianni Giovannini, ${ }^{2}$ Mauro Marchesi, ${ }^{1}$ for the Umbria PBM Group

To cite: Abraha I, Montedori A, Di Renzo GC, et al. Diagnostic, preventive and therapeutic evidence in obstetrics for the implementation of patient blood management: a systematic review protocol. BMJ Open 2018;8:e021322. doi:10.1136/ bmjopen-2017-021322

- Prepublication history for this paper is available online To view these files, please visit the journal online (http://dx.doi org/10.1136/bmjopen-2017021322).

Received 21 December 2017 Revised 18 May 2018 Accepted 26 July 2018
Check for updates

(C) Author(s) (or their employer(s)) 2018. Re-use permitted under CC BY-NC. No commercial re-use. See rights and permissions. Published by BMJ.

For numbered affiliations see end of article.

Correspondence to

Dr losief Abraha;

iosief_a@yahoo.it

\section{ABSTRACT}

Introduction Patientblood management (PBM) is defined as the application of evidence-based diagnostic, preventive and therapeutic approaches designed to maintain haemoglobin concentration, optimise haemostasis and minimise blood loss in an effort to improve patient outcome. We propose a protocol for the assessment of the evidence of diagnostic, preventive and therapeutic approaches for the management of relevant outcomes in obstetrics with the aim to create a framework for PBM implementation.

Methods and analysis Diagnostic, preventive and therapeutic tools will be considered in the gynaecological conditions and obstetrics setting (antenatal care, peripartum care and maternity care). For each condition, (1) clinical questions based on prioritised outcomes will be developed; (2) evidence will be retrieved systematically from electronic medical literature (MEDLINE, EMBASE, the Cochrane Library, Web of Science, and CINAHL); (3) quality of the reviews will be assessed using the AMSTAR (A Measurement Tool to Assess Systematic Reviews) checklist; quality of primary intervention studies will be assessed using the risk of bias tool (Cochrane method); quality of diagnostic primary studies will be assessed using QUADAS-2 (Quality Assessment of Diagnostic Accuracy Studies); (4) the Grading of Recommendations Assessment, Development and Evaluation method will be applied to rate the quality of the evidence and to develop recommendations.

Ethics and dissemination For each diagnostic, preventive or therapeutic intervention evaluated, a manuscript comprising the evidence retrieved and the recommendation produced will be provided and published in peer-reviewed journals. Ethical approval is not required.

\section{INTRODUCTION}

Patient blood management (PBM) is defined as the application of evidence-based diagnostic, preventive and therapeutic approaches

\section{Strengths and limitations of this study}

- The strength of the study is its comprehensive and systematic gathering of evidence concerning diagnostic, preventive and therapeutic means for the management of relevant outcomes in obstetrics to create a framework for patient blood management implementation

- For diagnostic evidence, we will use the QUADAS-2 (Quality Assessment of Diagnostic Accuracy Studies) instrument to assess the methodological quality.

- We will rate the evidence and formulate the recommendations based on the Grading of Recommendations Assessment, Development and Evaluation approach.

- Language bias may occur since only studies published in English or Italian will be included.

- Heterogeneity is expected due to characteristics of participants and types of interventions.

designed to maintain haemoglobin concentration, optimise haemostasis and minimise blood loss in an effort to improve patient outcome. ${ }^{1-4}$ The PBM approach is based on three pillars: (1) optimise erythropoiesis; (b) minimise blood loss and bleeding; and (c) optimise the physiological reserve of anaemia. ${ }^{5}$ To reach this aim, clinicians involved in the management of patients that require the administration of blood components need to highlight the concept that blood components are unique resources that should be used appropriately, should not be wasted and their administration should be performed within a of multidisciplinary, multimodal (eg, the application of a blood 
conservation programme that incorporates the aggressive management of preoperative anaemia and tolerance of perioperative anaemia as an important component ${ }^{6}$ ) and individualised strategy context. ${ }^{7-9}$

National and international guidelines have promoted and endorsed the application of PBM especially in the perioperative setting. ${ }^{10}$ For example, perioperative anaemia and the need for allogenic blood transfusions are associated with negative outcomes after major joint replacement. ${ }^{11}{ }^{12}$ The introduction of active blood management programmes, including measures to detect and treat anaemia several weeks before elective primary hip or knee replacement, generated significant improvements in outcomes such as lower allogeneic blood transfusion rates, shorter length of stay and a reduction of readmission rates. ${ }^{1}$

From pregnancy to the postpartum period including delivery, women may need different types of care in different settings (such as ambulatory during pregnancy, admission in hospital during labour). Indeed, pregnancy is characterised by physiological modifications in circulation, such as an increase in total volume, that is required for placental development and fetal growth. Hence, haemodilution and reduced blood viscosity due to the increase in plasma volume and red cell mass need to be taken into account during blood component management. In addition, compared with the generally transfused population, peripartum women are generally young $^{13}$ and the long-term consequences such as immunological effects can be critical. ${ }^{14}$

According to the WHO, causes of maternal mortality can be categorised as direct and indirect. While direct maternal mortality can be the result of complications or management of the pregnancy and delivery (such as pre-eclampsia/eclampsia, haemorrhage, puerperal sepsis, etc), indirect maternal mortality is defined as 'a pregnancy-related death in a mother with a pre-existing or newly developed health problem unrelated to pregnancy' (eg, cardiac disease, HIV/AIDS or chronic hypertension). Indirect causes are responsible for about one-fifth of severe maternal outcomes, $50 \%$ of which is represented by anaemia. ${ }^{15}$ Vogel et al found that risk of all perinatal mortality was significantly increased with placental abruption, ruptured uterus, systemic infections/sepsis, pre-eclampsia, eclampsia as well as severe anaemia. ${ }^{16}$ Postpartum haemorrhage (PPH) is among the leading causes of maternal mortality and morbidity during pregnancy worldwide. Allogenic blood transfusion is among the most common approaches used in obstetrics to treat PPH and there are sources that warn that rates of transfusion are increasingly being used during childbirth. ${ }^{17}$ Though the majority of deaths occur in low-income countries, ${ }^{19}$ recent reports indicate that there is an increasing trend in the incidence of $\mathrm{PPH}$ over time in Western countries. ${ }^{20}$ The use of allogenic blood transfusion should be supported by sound evidence, taking into account the link between transfusion and worsening of clinical outcomes and debated efficacy. ${ }^{21}{ }^{22}$ Hence, there is a need to revise the evidence of therapeutic and diagnostic interventions for relevant outcomes in obstetrics and gynaecology in order to produce PBM-based recommendations that are suitable for clinicians and patient decision makers at the local and national level.

The aim of the present protocol is to undertake a literature search of systematic reviews or primary studies regarding therapeutic, preventive and diagnostic interventions necessary to maintain haemoglobin concentration, optimise haemostasis and minimise blood loss in the context of obstetrics and gynaecology. In addition, the present study will provide a framework for adopting PBM through the development of clinical guidelines to assist clinicians, transfusionists, obstetricians and anaesthetists about appropriate care in gynaecology and obstetrics.

\section{METHODS}

For each diagnostic, preventive and therapeutic measure, the following steps will be considered:

a. Prioritising critical outcomes and formulating clinical questions;

b. Retrieving the evidence;

c. Developing recommendations.

\section{Prioritising critical outcomes and formulating clinical questions}

For each of the potential conditions within the aforementioned phases, the team will prioritise critical outcomes based on the Delphi method. First, a list of relevant outcomes will be submitted to a panel of experts in obstetrics, transfusion medicine, anaesthesiology, clinical epidemiology and public health for evaluation, discussion and rating. ${ }^{24}$ A maximum of three rounds of consultation will be performed depending on the variability in the ranking of the outcome. In case of large variability in the ranking of the outcomes, the results will be discussed with the panel members before the subsequent round. At the final stage, outcomes will be rated as: critical (score 7-9), important but not critical (score 4-6) or low importance (1-3).

Clinical questions that will take into account the diagnostic tool or the preventive and therapeutic interventions will be formulated based only on critical outcomes.

\section{Setting}

The interventions administered to avoid or treat outcomes will depend on the setting, the purpose of the intervention (in terms of prevention or treatment) and the status of the women: (1) antenatal care; (2) peripartum care; and (3) postpartum care within 6 weeks after delivery. The nature of the intervention will depend on the purpose for which it is recommended: (1) prevention or (2) treatment.

In addition, in each condition and setting, the evidence regarding potential diagnostic tools for which a recommendation might be necessary will be proposed. 


\section{Retrieving the evidence}

For evidence retrieval, we will consider first systematic reviews, and where the evidence is not sufficiently updated or when specific reviews are missing, we will produce new systematic reviews.

\section{Inclusion criteria for systematic reviews}

For each condition, to identify the abstracts of interest, we will prepare appropriate search strategies to be run in the following databases (see online supplemental file 1): PubMed, the Cochrane Library and Web of Science.

The following criteria will be considered for SRs (systematic reviews) inclusion: (1) a paper generally defined as a review; (2) any intervention that can be used to prevent or treat critical outcomes in women in gynaecological or obstetrics settings; (3) articles published in English or Italian; and (4) AMSTAR score $\geq 7$. Guidelines will be excluded but will be considered for reference checking to identify potentially relevant SRs.

Pairs of reviewers will independently screen titles, abstracts and full texts. Disagreement will be resolved by discussion and, if necessary, by a third independent reviewer. The process of published study selection will be presented in a Preferred Reporting Items for Systematic Reviews and Meta-Analyses flow diagram (figure 1).

The methodological quality of each SR will be assessed using the AMSTAR (A Measurement Tool to Assess Systematic Reviews) instrument. AMSTAR appraises the quality of reviews using the following 11 items: duplicate study selection and data extraction, comprehensive searching of the literature, provision of a list of included and excluded studies, provision of characteristics of included studies, assessment of methodological quality of included studies, appropriate methods for combining results of studies and for assessing publication bias and consideration of conflict of interest statement. ${ }^{25}$ Two review authors will independently evaluate the quality of the SRs and disagreement will be resolved by consensus.

\section{Inclusion criteria for primary studies}

For the efficacy reviews, we will identify and consider any comparative study, either randomised or non-randomised, that investigated any intervention to prevent and/or treat critical outcomes as appropriate. In general, priority will be given to randomised studies over non-randomised studies.

For diagnostic accuracy reviews, we will consider primarily cross-sectional studies that evaluated the accuracy of tests (such as rotational thromboelastography ${ }^{26}$ ) to diagnose outcomes of interest within the designated period.

\section{Data extraction and management}

Pairs of reviewers will perform data extraction from primary studies independently. Data will be extracted onto study-specific data extraction forms. Information

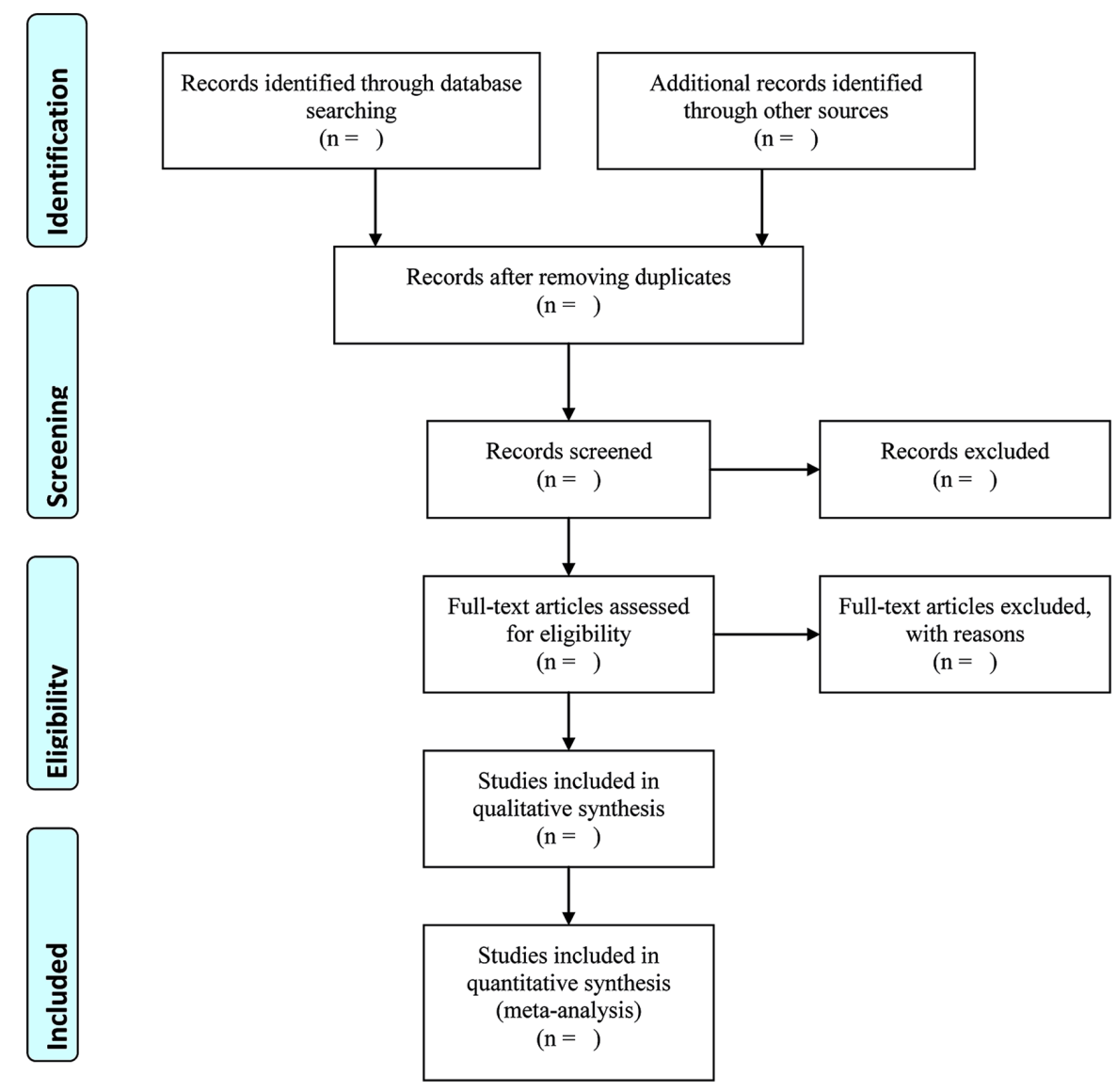

Figure 1 Study screening process. 
collected will include trial characteristics (year of publication, country of origin of the study, methodological quality items of the study), patients' characteristics (number of participants, age, gender), intervention characteristics, comparator characteristics, type of outcome and outcome measures. For diagnostic accuracy studies, the following data will be extracted: clinical features and settings in which the test has been developed, the index test and reference standard or comparator characteristics, description of the target condition.

\section{Assessing the methodological quality of the evidence}

For efficacy, evidence will be assessed using the Grading of Recommendations Assessment, Development and Evaluation (GRADE) approach. ${ }^{27}$ For each clinical question, evidence profiles based on the results of the treatment effect will be prepared. The following factors that may affect the rating of quality will be considered: (1) the study design and execution, (2) the consistency of results, (3) the directness of the evidence, (4) the precision of the estimate of the effect and (5) the likelihood of publication bias.

For the risk of bias (study design and execution), we will assess studies according to random sequence generation, ${ }^{28}$ allocation concealment, ${ }^{28}$ blinding of participants and personnel, ${ }^{28}$ blinding of outcome assessment, ${ }^{28}$ incomplete outcome data, ${ }^{29}{ }^{30}$ selective reporting ${ }^{31}$ and other potential items that can be a source of bias. Review authors will assign each study to one of the following categories: low risk, unclear risk and high risk.

Consequently, the body of evidence will be classified into four categories: (1) high (further research is very unlikely to change our confidence in the estimate of the effect), (2) moderate (further research is likely to have an important impact on our confidence in the effect and may change the estimate), (3) low (further research is very likely to have an important impact on our confidence in the estimate of effect and is likely to change the estimate) and (4) very low (any estimate of effect is very uncertain).

In case of non-randomised studies, the body of evidence will by default be rated as low but the quality can be upgraded based on the presence of the following three factors: (1) a strong or very strong association, (2) a doseeffect relationship and (3) all plausible confounding may be working to reduce the demonstrated effect or increase the effect if no effect was observed. ${ }^{32}$

For diagnostic evidence, we will use the QUADAS-2 (Quality Assessment of Diagnostic Accuracy Studies) instrument to assess the methodological quality. ${ }^{33}$ The QUADAS-2 instrument is made up of four domains: patient selection; index test; reference standard; and flow and timing. Each domain is assessed in terms of risk of bias, with the first three domains also considered in terms of applicability. ${ }^{34}$ Pairs of review authors will independently assess the quality that will be rated as 'yes', 'no' or 'unclear'.

\section{Assessment of heterogeneity}

For efficacy/safety evidence, we will assess heterogeneity according to the approach recommended by the Cochrane Handbook. ${ }^{35}$ Where a meta-analysis is possible with at least two studies, we will use the $\chi^{2}$ test and $\mathrm{I}^{2}$ statistic to assess heterogeneity. We will consider heterogeneity to be statistically significant if the $p$ value is less than 0.1 .

For diagnostic accuracy evidence, we will evaluate heterogeneity based on clinical factors, types of interventions, the characteristics of the index test and reference standard.

\section{Data synthesis}

For efficacy evidence, we will use risk ratios or ORs along with their $95 \%$ CI for binary outcome measures, whereas mean difference with $95 \% \mathrm{CI}$ will be used to estimate the summary effect for continuous outcome measures and, when data are measured on different scales, the standardised mean difference will be used. We will carry out data synthesis using Review Manager software (V.5). Depending on the expected level of heterogeneity between studies, we will use the random-effects model.

For diagnostic evidence, we will generate a $2 \times 2$ table of true positive cases, false positive cases, false negative cases and true negative cases. We will calculate sensitivities and specificities with $95 \%$ CIs for each study. We will perform meta-analyses by using the bivariate model. ${ }^{36}$ We will use STATA V.13 to generate parameter estimates (logit and variances) and will generate (1) the summary receiver operating characteristic curve, (2) the summary operating point, (ie, summary values for sensitivity and specificity), (3) a 95\% confidence region around the summary operating point and (4) a $95 \%$ prediction region.

\section{Developing recommendations}

The team will discuss and evaluate the net health benefit based on the anticipated balance of benefits and harms across all critical outcomes. For each clinical question, a Summary of Findings (SoF) table will be produced taking into account the gathered evidence. The SoF will summarise the quality of the evidence, the certainty about the balance of benefits versus harms, the similarity in patients' values and preferences and the costs of an intervention compared with the available alternatives. ${ }^{37}$

The strength of a recommendation will be categorised as 'strong' or 'weak'. It will be determined by the following factors: the quality of evidence, the balance between desirable effects and undesirable ones, the values and preferences and the resources and costs. The strength of a recommendation will be considered strong when the team is confident that the desirable effects of adherence to the recommendation outweigh the undesirable effects. High or moderate quality of evidence supports strong recommendations when this is also supported by other considerations such as the baseline risk of the population of interest, availability of the service and accessibility to care and costs. 
The strength of recommendation will be considered weak when the balance of benefit and harm is uncertain (quality of evidence is low or very low), or when values and preferences are uncertain or when much higher costs are envisaged..$^{38} 39$

\section{Patient and public involvement}

Patients and the public were not directly involved in the preparation of the present protocol.

\section{DISCUSSION}

'Blood transfusion' is generally considered as the administration of packed red cells or whole blood, ${ }^{40}$ platelets, ${ }^{41}$ fresh-frozen plasma ${ }^{42}$ or coagulation factors. In particular, allogenic blood transfusion has gained a central role in the management of a wide spectrum of medical and surgical diseases. However, recent progress in the identification and implementation of best transfusion practices on the basis of evidence-based systematic reviews suggests that, compared with a liberal allogenic blood transfusion policy, there was no evidence of negative consequences when following a restrictive blood transfusion policy. ${ }^{40}$ As PBM is being increasingly introduced in routine clinical practice,${ }^{10}{ }^{43-45}$ there is wide expectation that it will shape the practice of transfusion medicine, the modality of prescription, preparation and administration of blood components as well as the relationship between different disciplines. ${ }^{9}$ PBM brings a paradigm shift in the concept of blood components which should be considered not only an important resource but also a possible risk factor, with increases in costs, a sometimes limited availability: risky, costly, in limited supply, and their use can worsen negative patient outcomes. ${ }^{46}$ PBM aims to overcome the 'product-centred' concept of blood components and to have a 'patient-centred' approach which focusses on improving the health and well-being of the patient. ${ }^{46}$ In this patient-centred approach, it is required to set up a multiprofessional, multidisciplinary team by involving experts in transfusion medicine, anaesthetists and, depending on the context of the specialty, surgeons, orthopaedics or gynaecologists. Introducing transfusion practice improvement through the implementation of PBM can be an effective way of promoting high-value care by ameliorating patient outcomes, reducing blood product utilisation and product-related cost savings. ${ }^{47} 48$

All the key aspects of PBM are applicable also in the gynaecology and obstetrics setting to treat, prevent outcomes that require the management of anaemia, blood loss, optimise haemostasis and establish decision threshold for transfusion. ${ }^{18}$ A qualitative study that reviewed national and international guidelines for PBM in obstetrics identified important differences in recommendation for transfusion and PBM. The study emphasised that non-obstetrics guidelines were more likely to contain contemporary approaches to transfusion management than the obstetrics guidelines. The reason for variation may lie in the methods of guideline development, literature review and keenness to include evidence from non-obstetrics settings. These features will be taken into consideration to improve the quality of reporting of the present assessment.

One of the strengths of our study protocol is that we propose the use of the GRADE approach to evaluate the evidence retrieved in the electronic literature. GRADE offers a system of rating quality of evidence for systematic reviews and a method for grading the strength of recommendations in guidelines. GRADE allows the development of a wide range of clinical questions, including therapy, diagnosis as well as prevention. This method indicates a transparent way to frame the question after choosing and rating the outcomes that are considered critical for decision making. Once the evidence is retrieved, GRADE provides tools to rate the evidence by taking into account the risk of bias, the consistency of the body of the evidence, the precision of the effect estimate, any potential publication bias and the direct applicability of the body of the evidence to the patient population for which the recommendation is developed. The GRADE system suggests ways on how to incorporate evidence with considerations of values and preferences before providing recommendations. This approach has been successfully applied in several settings, one in which we have gained sufficient experience ${ }^{24} 3949$ to provide a framework to develop PBM recommendations in obstetrics and gynaecology.

As pointed out by Franchin and Muñoz, ${ }^{43}$ there are a number of initiatives that have been undertaken by the Italian National Blood Centre to promote the adoption of PBM in Italy, including the release of recommendations for the implementation of PBM in elective major orthopaedic surgery in adults. ${ }^{10}$

In a general context, the present study will be the first to highlight reviews that address these potential interventions and summarise their results for critical outcomes. At a regional level, the present initiative will be the first to contribute to the development of a framework for PBM recommendations in obstetrics.

\section{Ethics and dissemination}

A formal ethical approval will not be needed because the data used in this systematic review will not consider individual patient data and there will be no concerns about privacy. The results of the overviews of reviews or systematic reviews for each diagnostic, preventive or therapeutic interventions, a manuscript comprising the evidence retrieved and the recommendation produced will be provided and published in peer-reviewed journals and disseminated in conference presentations.

\section{Author affiliations}

${ }^{1}$ Centro Regionale Sangue, Servizio Immunotrasfusionale, Azienda 0spedaliera di Perugia, Perugia, Italy

${ }^{2}$ Health Planning Service, Regional Health Authority of Umbria, Perugia, Italy ${ }^{3}$ Clinica Ostetrica e Ginecologica, Policlinico, Università di Perugia, Perugia, Italy

${ }^{4}$ Ginecologia e Ostetricia, USL Umbria 2, Orvieto, Italy

${ }^{5}$ Servizio Immunotrasfusionale, USL Umbria 2, Foligno, Italy 
${ }^{6}$ Anestesia e Rianimazione, USL Umbria 2, Foligno, Italy

${ }^{7}$ Servizio Immunotrasfusionale, Azienda 0spedaliera Santa Maria di Terni, Terni, Italy

${ }^{8}$ Anestesia e Rianimazione, Azienda Ospedaliera Santa Maria di Terni, Terni, Italy

${ }^{9}$ Ginecologia e Ostetricia, Azienda Ospedaliera Santa Maria di Terni, Terni, Italy

${ }^{10}$ Terapia Intensiva e Rianimazione, USL Umbria 1, Perugia, Italy

${ }^{11}$ Ginecologia e Ostetricia, USL Umbria 1, Perugia, Italy

${ }^{12}$ Servizio Immunotrasfusionale, USL Umbria 1, Perugia, Italy

${ }^{13}$ Anestesia e Rianimazione, Azienda Ospedaliera di Perugia, Perugia, Italy

${ }^{14}$ Ginecologia e 0stetricia, Azienda Ospedaliera di Perugia, Perugia, Italy

Acknowledgements We are grateful to the Associazione Umbra Studio e Terapia Leucemie e Linfomi (A.U.L.L.) for covering the cost of publication and to Kathy Mahan for English editing of the protocol.

Collaborators Marina Onorato, Olivia Minelli, Nicola Albi, Barbara Luciani Pasqua, Cinzia Simeoni, Maria Isabella Bonacini, Francesco Cozzolino, Anna Chiara Lombardo, Claudia Moscetti, Massimiliano Orso, Paolo Eusebi

Contributors AM, IA and MM conceived the original idea of the study. IA, AM, GC, DR, PA, MM, DC, AG, GP, MC, CM, GN, EG, MP, LB, ST, GE, GG and MM drafted and approved the final version of the protocol. IA is the guarantor.

Funding This study is supported by the Regional Health Authority of Umbria Umbria Region.

Competing interests None declared.

Patient consent Not required.

Provenance and peer review Not commissioned; externally peer reviewed.

Open access This is an open access article distributed in accordance with the Creative Commons Attribution Non Commercial (CC BY-NC 4.0) license, which permits others to distribute, remix, adapt, build upon this work non-commercially, and license their derivative works on different terms, provided the original work is properly cited, appropriate credit is given, any changes made indicated, and the use is non-commercial. See: http://creativecommons.org/licenses/by-nc/4.0/.

\section{REFERENCES}

1. Kotzé A, Carter LA, Scally AJ. Effect of a patient blood management programme on preoperative anaemia, transfusion rate, and outcome after primary hip or knee arthroplasty: a quality improvement cycle. Br J Anaesth 2012;108:943-52.

2. Krishnamoorthy A, Hadi FA, Naidu A, et al. The modern implementation of patient blood management (PBM) in Malaysia: the use of intravenous iron in severe anaemia with iron deficiency - a case report. Med J Malaysia 2017;72:53-4.

3. SABM, 2010. Society for the advancement of blood management. http://www.sabm.org/about/ (accessed 21 Mar 2017).

4. Vaglio S, Gentili S, Marano G, et al. The Italian regulatory guidelines for the implementation of Patient Blood Management. Blood Transfus 2017;15:325-8.

5. Meier J, Gombotz H. Pillar III--optimisation of anaemia tolerance. Best Pract Res Clin Anaesthesiol 2013;27:111-9.

6. Moskowitz DM, McCullough JN, Shander A, et al. The impact of blood conservation on outcomes in cardiac surgery: is it safe and effective? Ann Thorac Surg 2010;90:451-8.

7. Farmer SL, Towler SC, Leahy MF, et al. Drivers for change: Western Australia Patient Blood Management Program (WA PBMP), World Health Assembly (WHA) and Advisory Committee on Blood Safety and Availability (ACBSA). Best Pract Res Clin Anaesthesiol 2013;27:43-58.

8. Shander A, Van Aken $\mathrm{H}$, Colomina MJ, et al. Patient blood management in Europe. Br J Anaesth 2012;109:55-68.

9. Isbister JP. The three-pillar matrix of patient blood management--an overview. Best Pract Res Clin Anaesthesiol 2013;27:69-84.

10. Vaglio S, Prisco D, Biancofiore G, et al. Recommendations for the implementation of a Patient Blood Management programme. Application to elective major orthopaedic surgery in adults. Blood Transfus 2016;14:23-65.

11. Husted H, Holm G, Jacobsen S. Predictors of length of stay and patient satisfaction after hip and knee replacement surgery: fasttrack experience in 712 patients. Acta Orthop 2008;79:168-73.

12. Spahn DR. Anemia and patient blood management in hip and knee surgery: a systematic review of the literature. Anesthesiology 2010;113:482-95.

13. Waters JH. Patient blood management in obstetrics. Int Anesthesiol Clin 2014;52:85-100.
14. Utter GH, Reed WF, Lee TH, et al. Transfusion-associated microchimerism. Vox Sang 2007;93:188-95.

15. Lumbiganon P, Laopaiboon M, Intarut N, et al. Indirect causes of severe adverse maternal outcomes: a secondary analysis of the WHO Multicountry Survey on Maternal and Newborn Health. BJOG 2014;121(Suppl 1):32-9.

16. Vogel JP, Souza JP, Mori R, et al. Maternal complications and perinatal mortality: findings of the World Health Organization Multicountry Survey on Maternal and Newborn Health. BJOG 2014;121(Suppl 1):76-88.

17. Knight M, Callaghan WM, Berg C, et al. Trends in postpartum hemorrhage in high resource countries: a review and recommendations from the International Postpartum Hemorrhage Collaborative Group. BMC Pregnancy Childbirth 2009;9:55.

18. Shaylor R, Weiniger CF, Austin N, et al. National and International Guidelines for Patient Blood Management in Obstetrics: A Qualitative Review. Anesth Analg 2017;124:216-32.

19. Sheldon WR, Blum J, Vogel JP, et al. Postpartum haemorrhage management, risks, and maternal outcomes: findings from the World Health Organization Multicountry Survey on Maternal and Newborn Health. BJOG 2014;121(Suppl 1):5-13.

20. Ford JB, Patterson JA, Seeho SK, et al. Trends and outcomes of postpartum haemorrhage, 2003-2011. BMC Pregnancy Childbirth 2015:15:334.

21. Shah A, Stanworth SJ, McKechnie S. Evidence and triggers for the transfusion of blood and blood products. Anaesthesia 2015;70:10-e3.

22. Carless PA, Henry DA, Carson JL, et al. Transfusion thresholds and other strategies for guiding allogeneic red blood cell transfusion. Cochrane Database Syst Rev 2010;10:Cd002042.

23. Guyatt GH, Oxman AD, Kunz R, et al. GRADE guidelines: 2. Framing the question and deciding on important outcomes. J Clin Epidemiol 2011;64:395-400.

24. Abraha I, Cruz-Jentoft A, Soiza RL, et al. Evidence of and recommendations for non-pharmacological interventions for common geriatric conditions: the SENATOR-ONTOP systematic review protocol. BMJ Open 2015;5:e007488.

25. Shea BJ, Grimshaw JM, Wells GA, et al. Development of AMSTAR: a measurement tool to assess the methodological quality of systematic reviews. BMC Med Res Methodol 2007;7:10.

26. Hunt $\mathrm{H}$, Stanworth $\mathrm{S}$, Curry N, et al. Thromboelastography (TEG) and rotational thromboelastometry (ROTEM) for trauma induced coagulopathy in adult trauma patients with bleeding. Cochrane Database Syst Rev 2015;2:CD010438.

27. Balshem $\mathrm{H}$, Helfand M, Schünemann $\mathrm{HJ}$, et al. GRADE guidelines: 3. Rating the quality of evidence. J Clin Epidemiol 2011;64:401-6.

28. Savović J, Jones H, Altman D, et al. Influence of reported study design characteristics on intervention effect estimates from randomised controlled trials: combined analysis of metaepidemiological studies. Health Technol Assess 2012;16:1-82.

29. Abraha I, Cherubini A, Cozzolino F, et al. Deviation from intention to treat analysis in randomised trials and treatment effect estimates: meta-epidemiological study. BMJ 2015;350:h2445.

30. Abraha I, Cozzolino F, Orso M, et al. A systematic review found that deviations from intention-to-treat are common in randomized trials and systematic reviews. J Clin Epidemiol 2017;84:37-46.

31. Macura A, Abraha I, Kirkham J, et al. Selective outcome reporting: telling and detecting true lies. The state of the science. Intern Emerg Med 2010;5:151-5

32. Guyatt GH, Oxman AD, Sultan S, et al. GRADE guidelines: 9. Rating up the quality of evidence. $J$ Clin Epidemiol 2011;64:1311-6.

33. Whiting PF, Rutjes AW, Westwood ME, et al. QUADAS-2: a revised tool for the quality assessment of diagnostic accuracy studies. Ann Intern Med 2011;155:529-36.

34. Eusebi P, Giannandrea D, Biscetti L, et al. Diagnostic utility of CSF $\alpha$-synuclein species in Parkinson's disease: protocol for a systematic review and meta-analysis. BMJ Open 2016;6:e011113.

35. Higgins JPT, Green S. Cochrane Handbook for systematic reviews of interventions version 5.1.0: The Cochrane Collaboration, 2011. (updated Mar 2011).

36. Reitsma JB, Glas AS, Rutjes AW, et al. Bivariate analysis of sensitivity and specificity produces informative summary measures in diagnostic reviews. J Clin Epidemiol 2005;58:982-90.

37. Agoritsas T, Merglen A, Heen AF, et al. UpToDate adherence to GRADE criteria for strong recommendations: an analytical survey. BMJ Open 2017;7:e018593.

38. Andrews JC, Schünemann HJ, Oxman AD, et al. GRADE guidelines: 15. Going from evidence to recommendation-determinants of a recommendation's direction and strength. $J$ Clin Epidemiol 2013:66:726-35. 
39. Abraha I, Rimland JM, Trotta F, et al. Non-Pharmacological Interventions to Prevent or Treat Delirium in Older Patients: Clinical Practice Recommendations The SENATOR-ONTOP Series. J Nutr Health Aging 2016;20:927-36.

40. Carson JL, Stanworth SJ, Roubinian N, et al. Transfusion thresholds and other strategies for guiding allogeneic red blood cell transfusion. Cochrane Database Syst Rev 2016;10:Cd002042.

41. Estcourt LJ, Birchall J, Allard S, et al. Guidelines for the use of platelet transfusions. Br J Haematol 2017;176:365-94.

42. Desborough $\mathrm{M}$, et al. Fresh frozen plasma for cardiovascular surgery. Cochrane Database Syst Rev 2015;7:CD007614.

43. Franchini M, Muñoz M. Towards the implementation of patient blood management across Europe. Blood Transfus 2017;15:292-3.

44. Guerra R, Velati C, Liumbruno GM, et al. Patient blood management in Italy. Blood Transfus 2016;14:1-2.
45. Meybohm P, Richards T, Isbister J, et al. Patient blood management bundles to facilitate implementation. Transfus Med Rev 2017;31:62-71.

46. Shander A, Hofmann A, Isbister J, et al. Patient blood management-the new frontier. Best Pract Res Clin Anaesthesiol 2013;27:5-10.

47. Leahy MF, Hofmann A, Towler S, et al. Improved outcomes and reduced costs associated with a health-system-wide patient blood management program: a retrospective observational study in four major adult tertiary-care hospitals. Transfusion 2017;57:1347-58.

48. Thakkar RN, Lee KH, Ness PM, et al. Relative impact of a patient blood management program on utilization of all three major blood components. Transfusion 2016;56:2212-20.

49. Abraha I, Trotta F, Rimland JM, et al. Efficacy of non-pharmacological interventions to prevent and treat delirium in older patients: a systematic overview. The SENATOR project ONTOP Series. PLOS One 2015;10:e0123090. 